\title{
A Finite Element Analysis Rupture Index (FEARI) as an Additional Tool for Abdominal Aortic Aneurysm Rupture Prediction
}

\author{
Barry J. Doyle ${ }^{1}$, Anthony Callanan ${ }^{1}$, Michael T. Walsh ${ }^{1}$, Pierce A. Grace ${ }^{1,2}$ and \\ Timothy M. McGloughlin ${ }^{1, *}$
}

${ }^{I}$ Centre for Applied Biomedical Engineering Research (CABER), Department of Mechanical and Aeronautical Engineering, and Materials and Surface Science Institute, University of Limerick, Ireland; ${ }^{2}$ Department of Vascular Surgery, HSE Midwestern Regional Hospital, Limerick, Ireland

\begin{abstract}
Currently, abdominal aortic aneurysms (AAAs), which are a permanent dilation of the aorta, are treated surgically when the maximum transverse diameter surpasses $5.5 \mathrm{~cm}$. AAA rupture occurs when the locally acting wall stress exceeds the locally acting wall strength. There is a need to review the current diameter-based criterion, and so it may be clinically useful to develop an additional tool to aid the surgical decision-making process. A Finite Element Analysis Rupture Index (FEARI) was developed.

Ten patient-specific AAAs were reconstructed, and the corresponding wall stress computed. Previous experimental work on determination of ultimate tensile strengths (UTS) from AAA tissue samples was implemented in this study. By combining peak wall stress along with average regional UTS, a new approach to the estimation of patient-specific rupture risk has been developed.

Ten cases were studied, all of which were awaiting or had previously undergone surgical AAA repair. A detailed examination of these ten cases utilising the FEARI analysis suggested that there was a possibility that some of the AAAs may have been less prone to rupture than previously considered.

It is proposed that FEARI, used alongside other rupture risk factors, may improve the current surgical decision-making process. The use of FEARI as an additional tool for rupture prediction may provide a useful adjunct to the diameter-based approach in surgical decision-making.
\end{abstract}

Keywords: Aneurysm, rupture, prediction, stress, strength.

\section{INTRODUCTION}

Cardiovascular disease is a leading cause of death in the Western world. Aneurysms, which are dilations or widening of blood vessels, form a significant portion of these deaths. The healthy infrarenal abdominal aorta diameter ranges from $15 \mathrm{~mm}$ to $24 \mathrm{~mm}$ for most elderly men [1], with abdominal aortic aneurysms (AAAs) defined as an aorta with an infrarenal diameter 1.5 times that of the normal aortic diameter [2]. The actual mechanisms resulting in AAA formation are still not clearly understood. It is believed that these aneurysms form due to alterations of the connective tissue in the aortic wall. This degradation of the aortic wall can be attributed to risk factors such as tobacco smoking, sex, age, hypertension, chronic obstructive pulmonary disease, hyperlipidaemia, and family history of the disorder [1]. Increased screening of subjects and improved imaging technologies, have given rise to increased detection of AAAs. Approximately 150,000 new cases are diagnosed each year in the USA [3-5], resulting in 15,000 deaths per year due to AAA rupture [6].

*Address correspondence to this author at the MSG-014, MSSI Building, University of Limerick, Limerick, Ireland; Tel: +353 61 202369; Fax: +353 61 202944; E-mail: tim.megloughlin@ul.ie
Currently, the rupture risk of AAAs is regarded as a continuous function of aneurysm size. Traditionally, surgeons operate once the AAA exceeds a transverse diameter of $50 \mathrm{~mm}$ [7-10], but previous work has shown that AAAs smaller than $50 \mathrm{~mm}$ can also rupture $[11,12]$. It is believed by many researchers that there is a need to review the determination of the timing of surgical intervention based solely on aneurysm diameter, and consider the inclusion of other relevant risk factors.

It is known that AAA rupture occurs when the locally acting wall stress exceeds the locally acting wall strength. Therefore, the AAA tissue strength must play an equal role to AAA wall stress in determining failure. A region of AAA wall that is under elevated wall stress may also have high wall strength, thus equalising its rupture potential. Many researchers have proposed alternative methods of determining AAA rupture potential. These additional risk factors could, for example, include: AAA wall stress [9, 10]; AAA expansion rate $[6,13,14]$; degree of asymmetry [15-17]; presence of intraluminal thrombus (ILT) [18] which is a fibrin structure incorporated with blood cells, platelets, blood proteins, and cellular debris, and are found in most AAAs; a rupture potential index (RPI) $[19,20]$; biomechanical factors and computer analysis [6]; growth of thrombus [21]; geomet- 
rical parameters [22]; and also a method of determining AAA growth and rupture based on a mathematical model [23].

The purpose of this study is to examine the use of a new additional tool to assist in the assessment of AAA rupture risk. This new approach focuses on a combination of the finite element method (FEM) coupled with published ultimate tensile strength (UTS) data from AAA tissue reported by previous researchers $[13,24,25]$. The FEM is a numerical technique widely used in engineering that divides complex 3D structures into small areas called elements, for which the stress distribution can be more easily studied. This new approach which we have described as the Finite Element Analysis Rupture Index (FEARI), may be clinically useful in aiding surgeons as to the most appropriate time to surgically intervene, and may serve as a useful adjunct to maximum diameter.

\section{MATERIALS AND METHODS}

\section{Study Subjects and Reconstructions}

Computed tomography (CT) scan data was obtained for 10 patients (male, $n=6$; female, $n=4$ ). These patient scans were obtained from the Midwestern Regional Hospital, Limerick, Ireland, and the University of Pittsburgh Medical Centre, Pittsburgh, PA, USA. All ten patients were awaiting or had received AAA repair at the time of CT scan, as AAA diameters had reached or exceeded the current $5.0 \mathrm{~cm}$ threshold for repair. 3D reconstructions were performed using the commercial software Mimics v10.0 (Materialise, Belgium). This reconstruction technique was validated with previous work performed by our group [26, 27]. The iliac arteries were omitted from the reconstruction as they have been shown not to significantly alter the resulting wall stress distributions [10]. Patient-specific wall thickness was obtained using the equation proposed by $\mathrm{Li}$ and Kleinstreuer [28] shown in Eqn. 1, where $t$ is wall thickness and Dia ${ }_{\max }$ is the maximum diameter. The ILT was also included in the reconstructions as this structure has been shown to reduce wall stress [18]. The models developed by this method formed the basis for the FEM stress analysis. The pre-operative details of the ten cases examined can be seen in Table $\mathbf{1}$.

$$
\mathrm{t}=3.9\left(\frac{\mathrm{Dia}_{\max }}{2}\right)^{-0.2892}
$$

\section{Wall Stress Analysis}

The ten $3 \mathrm{D}$ reconstructions were imported into the commercial finite element solver ABAQUS v6.7 (Dassault Systemes, SIMULIA, R.I., USA) for stress analysis. In order to simulate in vivo wall stress in the AAA wall, realistic boundary conditions were applied to each model. The AAA wall was modelled as a homogenous isotropic hyperelastic material using the finite strain constitutive model proposed by Raghavan and Vorp [8]. These material properties have been utilised in many previous stress analysis studies $[9,10,17$, $18,29,30-35]$. The aorta is also known to be nearly incompressible with a Poisson's ratio of 0.49 . The ILT was modelled as a hyperelastic material using the material characterisation derived from 50 ILT specimens from 14 patients performed by Wang et al. [36]. Each AAA was constrained in the proximal and distal regions to simulate tethering to the aorta at the renal and iliac bifurcations. The blood pressure within the AAA acts on the luminal contour of the ILT and therefore, pressure was applied to the inner surface of the computational AAA model. A static peak systolic pressure of $120 \mathrm{mmHg}(16 \mathrm{KPa})$ was used, as employed in most AAA stress analyses $[15,22,33,37,38]$. It is known that patientspecific blood pressures may be higher than $120 \mathrm{mmHg}$, but for the purpose of this study a standard value was more appropriate so as to eliminate some of the unknown variables in the analysis. The shear stress induced by blood flow was neglected in this study, although the effects of blood flow have been shown to reduce wall stress in idealised AAA models [16]. Residual stresses that may exist within the aortic wall in vivo and tethering forces on the posterior surface caused by the lumbar arteries were also neglected.

Once adequate boundary conditions were applied to each model, a mesh was generated on each AAA. Mesh independ-

Table 1. Pre-Operative Patient Details for Each Study Subject. Note that $\varnothing$ is Diameter

\begin{tabular}{|c|c|c|c|c|c|c|}
\hline Patient & Sex & $\begin{array}{c}\text { Max Ø } \\
(\mathbf{c m})\end{array}$ & $\begin{array}{c}\text { Wall Thickness } \\
\text { (mm) }\end{array}$ & $\begin{array}{c}\text { AAA Length } \\
\text { (cm) }\end{array}$ & $\begin{array}{c}\text { AAA Volume } \\
\qquad\left(\mathrm{cm}^{3}\right)\end{array}$ & $\begin{array}{l}\text { ILT Volume } \\
\quad\left(\mathrm{cm}^{3}\right)\end{array}$ \\
\hline 1 & Male & 5.1 & 1.53 & 13.2 & 176.7 & 54.1 \\
\hline 2 & Male & 5.75 & 1.48 & 11.2 & 192.4 & 122.8 \\
\hline 3 & Male & 5.85 & 1.47 & 11.6 & 194.9 & 96.8 \\
\hline 5 & Male & 5.9 & 1.47 & 12.8 & 220.9 & 116.8 \\
\hline 6 & Male & 7.4 & 1.37 & 14.3 & 311.6 & 147.6 \\
\hline 7 & Female & 5.3 & 1.51 & 10.5 & 137.9 & 82.5 \\
\hline 8 & Female & 6.2 & 1.48 & 9.7 & 61.6 & 29.1 \\
\hline
\end{tabular}


ence was performed for all AAA models in order to determine the optimum number of elements, and therefore, the optimum mesh. Mesh independence was performed by increasing the number of elements in the mesh until the difference in peak stress was less than $2 \%$ of the previous mesh $[17,18,34,38]$. This method of determining wall stress distributions has been previously shown to be the most effective in computing accurate results compared with other approaches [34].

\section{FEARI}

The Finite Element Analysis Rupture Index (FEARI) is defined by Eqn. 2. In this equation, the peak wall stress is computed using the FEM, whereas, the wall strength values are obtained from previous research on experimental testing of AAA wall specimens [13, 24, 25].

$$
F E A R I=\frac{\text { FEA Wall Stress }}{\text { Experimental Wall Strength }}
$$

Eqn. 2

This equation is based on the simple engineering definition of material failure, that is, failure will occur when the stress acting on the material exceeds the strength of the material. This index then returns a value ranging from 0 to 1 , where 0 indicates a very low rupture potential, and a value close to 1 indicates a very high rupture potential.

In order to determine strength values for the AAA wall, the previous research of both Raghavan et al. [24, 25] and Thubrikar et al. [13] were analysed. These publications are the most detailed reports of experimental uniaxial testing of AAA tissue. Raghavan et al. [24] tensile tested 52 specimens of AAA tissue and found that the average UTS of this aneurysmal tissue was $0.942 \mathrm{MPa}$. Thubrikar et al. [13] later segmented AAAs into posterior, anterior and lateral regions and tensile tested 49 tissue specimens. Average regional UTS values were shown to be $0.46 \mathrm{MPa}, 0.45 \mathrm{MPa}$ and 0.62 $\mathrm{MPa}$, respectively. Raghavan et al. [25] subsequently furthered their work, and also divided each AAA into regional sections and tested 48 samples. They showed that the UTS of AAA tissue can range from $0.336-2.35 \mathrm{MPa}$. They also reported that the regional variations in UTS for the anterior, posterior, left and right regions, were 1.099 $\mathrm{MPa}, 1.272$ $\mathrm{MPa}, 1.217 \mathrm{MPa}$ and 1.224 $\mathrm{MPa}$, respectively.

By combining all the previously published experimental data $[13,24,25]$, average regional UTS values for the four main regions of the AAA could be obtained. By subdividing the AAA into a further four sections, eight in total, more regionally accurate strength estimates were obtained, thus allowing FEARI values to be calculated. The method of dividing each AAA into regions is shown in Fig. (1), with the resulting UTS values for the varying regions shown in Table 2.

Once the combined UTS values of AAA tissue were calculated, these values could be coupled with the computed wall stress results from the FEM. By recording the location of peak stress in each AAA model, the area can also be assigned a regional UTS value. FEARI was then computed for each of the ten cases. Statistical analysis was performed on all results using a Pearson's correlation, with $P<0.05$ accepted as significant.
Table 2. Regional UTS Values Obtained by Combining and Averaging Previous Experimental Data [13, 24, 25]

\begin{tabular}{|c|c|}
\hline AAA Region & UTS (MPa) \\
\hline \hline Anterior & 0.7744 \\
\hline Posterior & 0.8658 \\
\hline Left & 0.9221 \\
\hline Right & 0.9187 \\
\hline Anterior/Left & 0.8482 \\
\hline Anterior/Right & 0.8465 \\
\hline Posterior/Left & 0.8939 \\
\hline Posterior/Right & 0.8922 \\
\hline
\end{tabular}

\section{RESULTS}

\section{Wall Stress Results}

The finite element analyses using ABAQUS v6.7 produced detailed stress distributions on each of the models under the pressure loading [19]. The von Mises stress is a stress index especially suited for failure analysis, as stress is a tensor quantity with nine components, with the von Mises stress being a combination of these components. By observing the stress distributions, it was noted that the regions of elevated and peak wall stresses occurred at inflection points on the AAA surface, and not at regions of maximum diameter. This observation was also observed by previous researchers in idealised models, both experimentally [39] and numerically $[15,40]$ and also in realistic models $[17,34]$. Inflection points are defined as points on the AAA surface at which the local AAA wall shape changes from concave outward to concave inward [15]. The peak wall stresses found in this study ranged from $0.3167-1.282 \mathrm{MPa}$, with a mean \pm standard deviation of $0.6201 \pm 0.2836 \mathrm{MPa}$. The results of the computational stress analysis, along with the maximum diameter and UTS for each case can be seen in Table 3. There was no statistical significance between peak wall stress and any geometrical parameters analysed here. There was however a significant relationship between both FEARI and maximum diameter $(P=0.043)$, and FEARI and AAA volume $(P=0.036)$.

\section{FEARI Results}

Once all peak wall stresses, locations of peak wall stress, and corresponding regional UTS values were obtained and grouped, FEARI results using Eqn. 2 could be calculated. Table 4 and Fig. (2) show the resulting FEARI values with the corresponding maximum diameters of each patient. In this figure, an indication of the possible rupture risk using the FEARI analysis of each patient is shown. An example calculation of this index for Patient 9 can be seen below.

$$
F E A R I=\frac{\text { FEA Wall Stress }}{\text { Experimental Wall Strength }} \quad \text { Eqn. } 2
$$

Where, peak FEA wall stress is $0.5263 \mathrm{MPa}$, and this peak stress occurred in the anterior region of the AAA. 


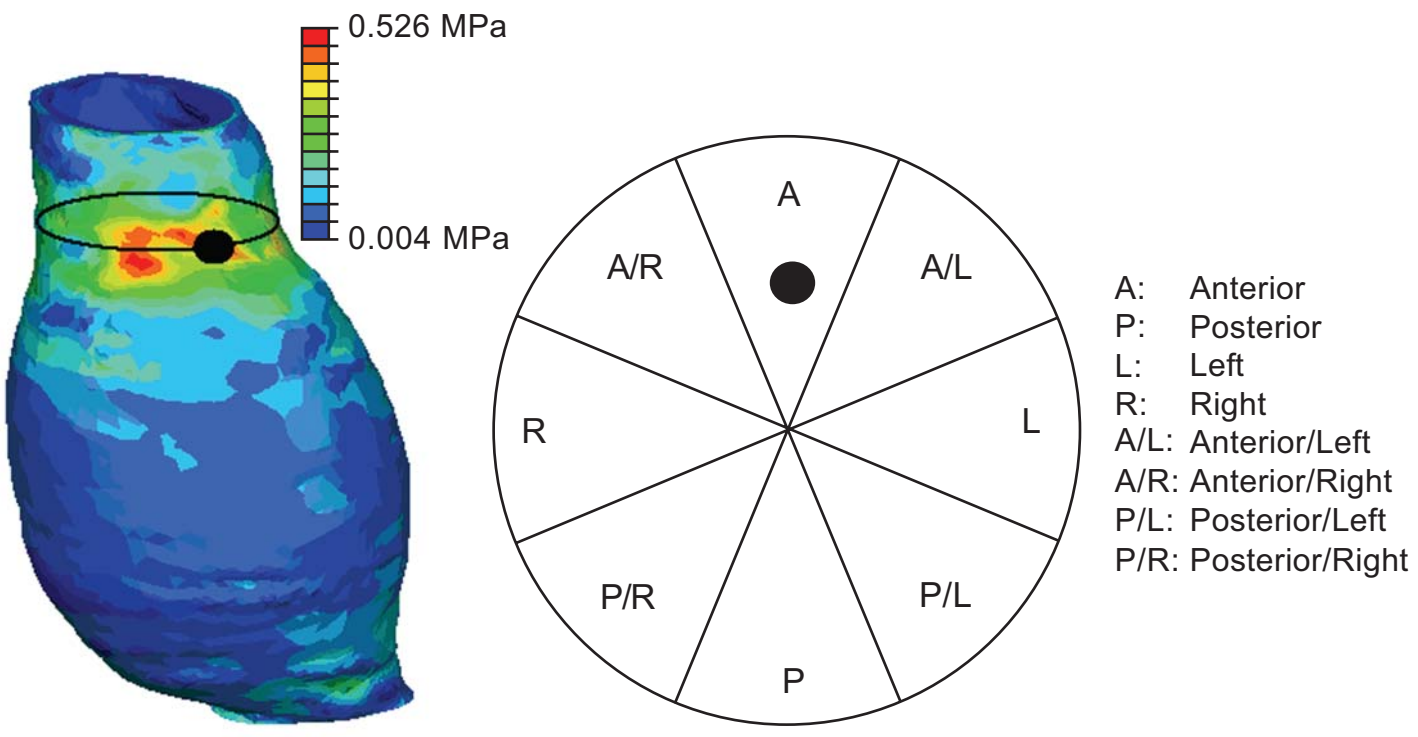

Fig. (1). Illustration showing a representative AAA stress distribution for Patient 9 (ellipse passes through region of peak stress, indicated by black circle) and how each AAA model was segmented in order to determine the corresponding UTS value for the particular region of peak stress. In this case, peak stress occurs on the anterior wall of the AAA. AAA model shown in the anterior view.

Table 3. Maximum Diameter, FEA Computed Peak Wall Stress, Location of Peak Wall Stress, and UTS of Peak Stress Region in All Ten Cases Examined. Wall Thickness was Incorporated in Peak Wall Stress Calculations

\begin{tabular}{|c|c|c|c|c|}
\hline Patient & Max Diameter (cm) & Peak Wall Stress (MPa) & Location & UTS (MPa) \\
\hline \hline $\mathbf{1}$ & 5.1 & 0.4291 & Anterior & 0.7744 \\
\hline $\mathbf{2}$ & 5.75 & 0.3167 & Left & 0.9221 \\
\hline $\mathbf{3}$ & 5.85 & 0.4346 & Anterior/Right & 0.8465 \\
\hline $\mathbf{4}$ & 5.0 & 0.6641 & Anterior/Right & 0.8465 \\
\hline $\mathbf{5}$ & 5.9 & 0.5866 & Anterior/Right & 0.8465 \\
\hline $\mathbf{6}$ & 7.4 & 0.707 & Anterior/Right & 0.9187 \\
\hline $\mathbf{7}$ & 5.3 & 0.4 & Anterior & 0.7744 \\
\hline $\mathbf{8}$ & 6.2 & 1.282 & Anterior & 0.7744 \\
\hline $\mathbf{9}$ & 6.5 & 0.5263 & Posterior & 0.8658 \\
\hline
\end{tabular}

Therefore, the UTS of the peak stress region is $0.7744 \mathrm{MPa}$ using Table 2. Eqn. 2 now becomes Eqn. 3.

$$
\text { FEARI }=\frac{0.5263}{0.7744}=0.6796
$$

Eqn. 3

The resulting FEARI for Patient 9 is 0.6796 , representing a possible $68 \%$ chance of rupture.

\section{DISCUSSION}

Considering the high level of mortality associated with AAA rupture, improved surgical decision tools may lead to better clinical outcomes. At present, the timing of intervention is determined on the basis of maximum diameter alone. However, it was reported that AAAs with diameters $<5.0 \mathrm{~cm}$ may be safe from rupture, and should be rigorously monitored [1]. The results presented here suggest that the inclusion of the FEARI monitoring approach to AAA management may be useful in the assessment of all size AAAs, rather than repairing the aneurysm based on maximum diameter alone.

It is known that AAA rupture occurs when the locally acting wall stress exceeds the locally acting wall strength, and thus the inclusion of wall strength as a rupture parameter may also be useful. The FEARI approach presented here utilises previously published experimental data from tissue specimens $[13,24,25]$ which were applied to establish FEARI results. The wall stress was computed using the commercial FEA solver, ABAQUS v6.7, from which, peak 
Table 4. Resulting FEARI Values Compared to the Corresponding Maximum Diameters for each AAA Studied

\begin{tabular}{|c|c|c|}
\hline Patient & $\begin{array}{c}\text { Maximum Diameter } \\
(\mathbf{c m})\end{array}$ & FEARI \\
\hline \hline 1 & 5.1 & 0.5541 \\
\hline 2 & 5.75 & 0.3435 \\
\hline 3 & 5.85 & 0.5133 \\
\hline 4 & 5.0 & 0.7845 \\
\hline 5 & 5.9 & 0.6929 \\
\hline 6 & 7.4 & 0.7696 \\
\hline 7 & 5.3 & 0.4725 \\
\hline 8 & 6.2 & 1.6554 \\
\hline 9 & 6.5 & 0.6796 \\
\hline 10 & 9.0 & 0.9876 \\
\hline
\end{tabular}

wall stress values and the location of peak stress could be recorded for each case. Convergence studies were also performed on each model to establish confidence in the finite element mesh size and accuracy of the results [17, 18, 34, 38]. Patient-specific wall thickness values were applied to each case using the equation proposed by $\mathrm{Li}$ and Kleinstreuer [28] to provide more patient-specific results. Peak stresses varied throughout the ten cases, and were independent of the maximum diameter of the AAA. There was no statistical significance between peak wall stress and any of the measured parameters (maximum diameter, $P=0.227$; sex, $P=0.404$; AAA volume, $P=0.936$; AAA length, $P=0.501$; ILT volume, $P=0.247$; wall thickness, $P=0.375$; location of peak stress, $P=0.54)$. FEARI results were also statistically compared with the same parameters. Of these comparisons, FEARI was statistically significant with maximum diameter $(P=0.043)$ and AAA volume $(P=0.036)$, whereas, there was no significance with other parameters (sex, $P=0.961$; AAA length, $P=0.804$; ILT volume, $P=0.881$; wall thickness, $P=0.056$; location of peak stress, $P=0.755)$. Significance was obtained when $P<0.05$. These results alone suggest that other rupture indicating parameters should be included in the surgical decision-making process. From the ten cases examined, all AAAs experienced peak wall stress at areas where the ILT was regionally thinner, therefore supporting the hypothesis that ILT reduces wall stress by acting like a "mechanical cushion" $[18,19,38]$ for the AAA wall. Another interesting observation relates to Patient 8 . In this case, the AAA geometry had a rapid change in diameter from $33.4 \mathrm{~mm}$ to $61.4 \mathrm{~mm}$ over $12.5 \mathrm{~mm}$. This sudden change in geometry resulted in a large peak stress of $1.282 \mathrm{MPa}$, which is approximately $60 \%$ higher than the similarly sized-diameter AAA of Patient 9.

A FEARI value was determined for each case from Eqn. 2. A FEARI value close to or above 1, suggests that the AAA may be a high rupture-risk AAA, and should be repaired immediately. FEARI values closer to 0 , on the other hand, would indicate that the risk of rupture is low, and should be monitored closely through regular ultrasound or CT scanning. All ten cases examined had reached or exceeded the current $5.0 \mathrm{~cm}$ threshold for surgical repair. At the time of this study, all cases were awaiting or had undergone traditional open repair or endovascular aneurysm repair (EVAR). The peak stresses show that smaller AAAs can have higher peak wall stresses than larger AAAs (see Table 3). Wall stress is closely related to geometrical parameters, with highly stressed areas occurring at regions of inflection on the surface of the AAA. Tortuous and irregularly shaped, or asymmetric, AAAs can experience many regions of inflection, and thus, many regions acting as stress raisers on the surface.

Although FEARI results presented here are preliminary, in that they currently use experimental data from other researchers $[13,24,25]$, the approach may be clinically useful. In order to gauge the effect of the UTS on the resulting FEARI, the "worst case scenario" in terms of UTS was examined. Raghavan et al. [25] reported that the UTS of AAA tissue can range from $0.336-2.35 \mathrm{MPa}$, and therefore, this minimum strength of $0.336 \mathrm{MPa}$ was applied to our FEARI equation. The results can be seen in Fig. (3). This resulted in much higher rupture potentials for all ten cases, and suggested that all patients were suitable for immediate repair. Although this minimum UTS value was recorded, only 8 of the 48 specimens tested by Raghavan et al. [25] resulted in UTS values below $0.5 \mathrm{MPa}$, with $0.336 \mathrm{MPa}$ being the absolute minimum displayed by any specimen. Therefore, to assume that all AAAs, in all regions experience this low failure stress would appear to be overly conservative.

It is proposed that FEARI could serve as a useful adjunct to diameter-based surgical decision making. Diameter, and ultimately size, of the AAA is an obvious concern for the clinician, and must remain a consideration. The overall geometry of the aneurysm should also play a role. Asymmetry has been shown to affect wall stress in idealised AAA models, both numerically $[15,16,40]$ and experimentally[39], and may also affect realistic cases [17]. Other researchers have proposed the Rupture Potential Index (RPI) [20] which uses a statistical modelling technique with the inclusion of factors such as age, gender, family history, smoking status, among others, to deduce patient-specific wall strength [41]. This RPI approach uses a more theoretical method of calculating wall strength, compared to the experimental approach of FEARI, and combining the two approaches may lead to improved predictions. Ultimately, the decision to surgically intervene may include a combination of factors including diameter, asymmetry, RPI and FEARI, along with clinical experience, and may determine the most suitable approach to a particular AAA.

The use of fluid-structure interaction (FSI) software, as opposed to the current static solid-mechanics approach may reduce peak stresses [16]. The effect of varying blood pressure may also alter results. Blood pressure can change over time, often as a result of stress or exercise. Increasing or decreasing the applied pressure to the stress analyses would increase or decrease the FEARI results respectively. Monitoring a patients' blood pressure over the course of a day may allow a more accurate loading condition to be developed, and thus provide more accurate patient-specific FEARI 


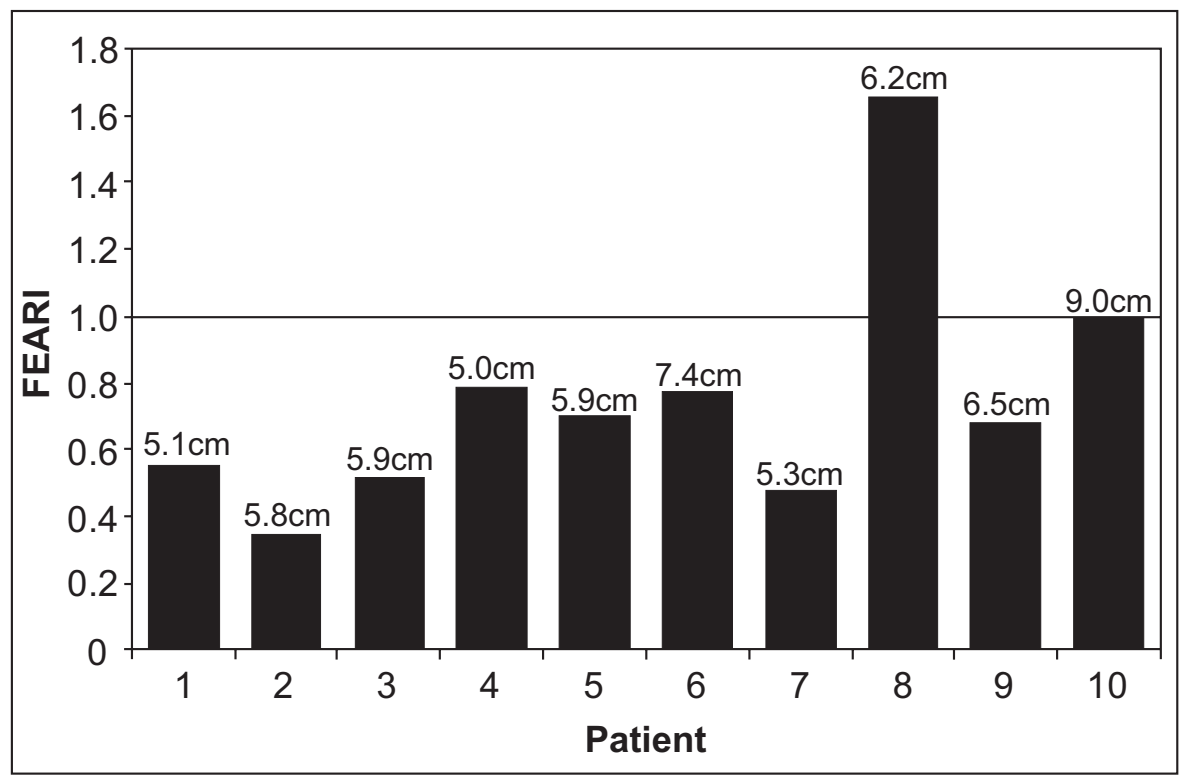

Fig. (2). Graph displaying FEARI results for all ten cases. Horizontal line indicates possible AAA rupture based on the FEARI model. Diameters of each case are presented also, indicating how diameter is not related to FEARI.

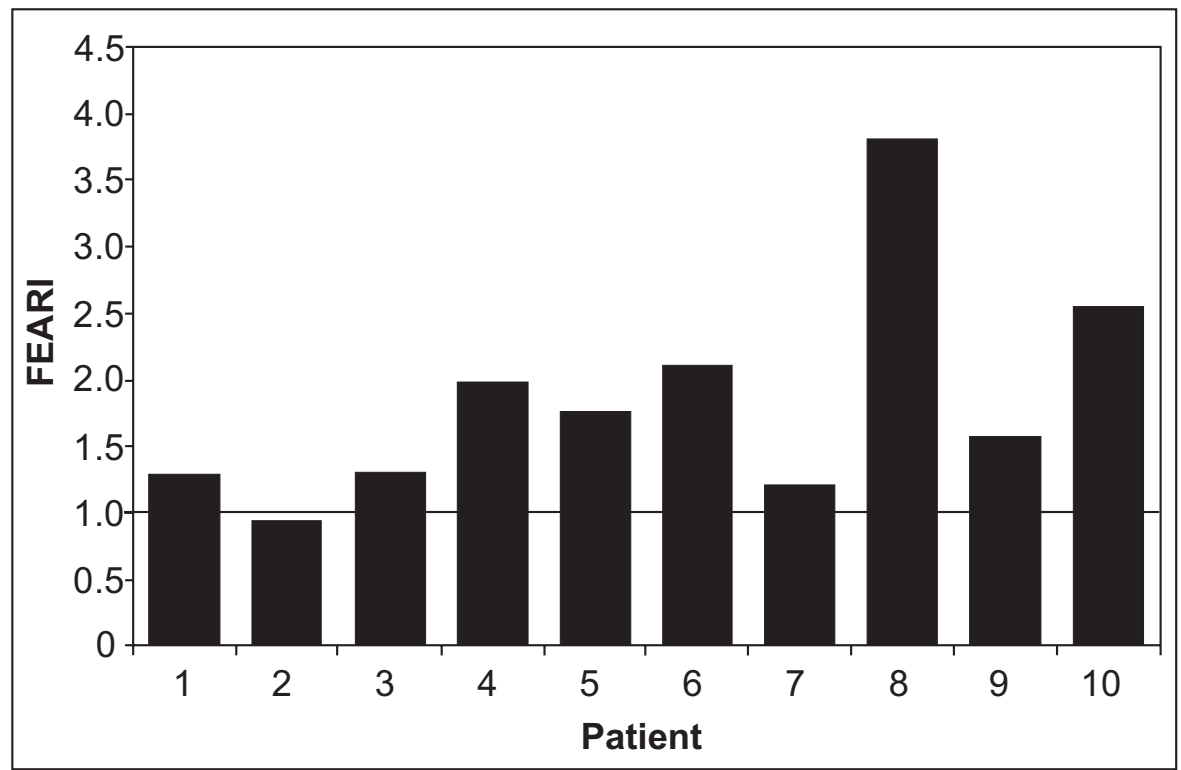

Fig. (3). FEARI results for the "worst case scenario" of extremely low failure strengths of the AAA tissue. Horizontal line indicates possible AAA rupture using the FEARI model.

results. It is known that the realistic AAA has a non-uniform wall thickness [25] and also non-uniform material properties due to regions of calcifications [15] which can lead to alterations in stress distributions [33]. The refinement of the technique used to compute wall stress may therefore produce more realistic FEARI results even when considering the "worst case scenario" regarding tissue strength. Along with these limitations in computing the peak wall stress, further experimental testing of AAA tissue is necessary. Both Raghavan et al. [24, 25] and Thubrikar et al. [13] recorded varying UTS values for the differing regions of the AAA, and therefore, a more widespread study on the UTS of AAA tissue is required. Collation of data from other centres worldwide, would lead to a much larger database of tissue strength, which could then be averaged over the population. Biaxial tensile testing of tissue is also necessary. It has been reported that AAA tissue is anisotropic, and that $3 \mathrm{D}$ multiaxial mechanical evaluation would allow for more appropriate modelling of aneurysmal tissue [42]. There have been recent reports on the use of anisotropic material data in wall stress analyses which may also yield better results [43]. By actually measuring AAA tissue harvested from unruptured AAAs during surgery, tensile strength data can be gathered. By expanding the database to many institutions, larger populations can be covered, thus increasing the effectiveness of the AAA strength database. This may lead to a more accurate FEARI measure in order to improve the assessment of patients with AAAs. 


\section{CONCLUSIONS}

FEARI may be clinically useful due to the simplicity of the approach. Rupture occurs when the AAA tissue cannot withstand the locally acting wall stress exerted, and therefore, tissue strength must be considered when assessing AAA rupture potential. Peak wall stresses were computed, along with the location, and therefore UTS, of peak wall stress region. FEARI results indicate that surgery may not be necessary for all cases, but rather continued monitoring may suit particular patients. It is proposed to couple FEARI together with diameter and other important factors in AAA assessment to allow the clinician a greater understanding of the severity of an individual AAA before deciding on surgical intervention. This preliminary study of a FEARI suggests that further work into this approach may yield more accurate results, and may provide a useful adjunct to the diameterbased approach in surgical decision-making.

\section{ACKNOWLEDGEMENTS}

The authors would like to thank (i) the Irish Research Council for Science, Engineering and Technology (IRCSET) Grant RS/2005/340 (ii) Grant \#R01-HL-060670 from the US National Heart Lung and Blood Institute (iii) the Department of Vascular Surgery in the Midwestern Regional Hospital, Ireland, in particular, Mr. Eamon Kavanagh, Mr. Paul Burke and Prof. Pierce Grace (iv) Aidan Cloonan for his assistance with the numerical analyses (iv) Prof. David A. Vorp from the Centre for Vascular Remodelling and Regeneration, University of Pittsburgh and (v) Michel S. Makaroun, MD, Department of Surgery, University of Pittsburgh.

\section{REFERENCES}

[1] Sakalihasan N, Limet R, Defawe OD. Abdominal aortic aneurysm. Lancet 2005; 365: 1577-89.

[2] Johnston KW, Rutherford RB, Tilson MD, Shah DM, Hollier L, Stanley JC. Suggested standards for reporting on arterial aneurysms. Subcommittee on reporting standards for arterial aneurysms, Ad hoc committee on reporting standards, Society for Vascular Surgery and North American Chapter, International Society for Cardiovascular Surgery. J Vasc Surg 1991; 13: 452-8.

[3] Vorp DA. Biomechanics of abdominal aortic aneurysm. J Biomech 2007; 40: 1887-902.

[4] Ouriel K, Green RM, Donayre C, Shortell CK, Elliott J, DeWesse JA. An evaluation of new methods of expressing aortic aneurysm size: relationship to rupture. J Vasc Surg 1992; 15: 12-20.

[5] Bengtsson H, Sonesson B, Bergqvist D. Incidence and prevalence of abdominal aortic aneurysms, estimated by necroscopy studies and population screening by ultrasound. Ann NY Acad Sci 1996; 800: $1-24$.

[6] Kleinstreuer C, Li Z. Analysis and computer program for rupturerisk prediction of abdominal aortic aneurysms. Biomed Eng Online 2006; 5: 19

[7] Sayers RD. Aortic aneurysms, inflammatory pathways and nitric oxide. Ann R Coll Surg Eng 2002; 84: 239-46.

[8] Raghavan ML, Vorp DA. Toward a biomechanical tool to evaluate rupture potential of abdominal aortic aneurysm: identification of a finite strain constitutive model and evaluation of its applicability. J Vasc Surg 2000; 33: 475-82.

[9] Fillinger MF, Raghavan ML, Marra SP, et al. In vivo analysis of mechanical wall stress and abdominal aortic aneurysm rupture risk. J Vasc Surg 2002; 36: 589-97.

[10] Fillinger MF, Marra SP, Raghavan ML, et al. Prediction of rupture risk in abdominal aortic aneurysm during observation: wall stress vs. diameter. J Vasc Surg 2003; 37: 724-32.

[11] Nicholls SC, Gardner JB, Meissner MH, et al. Rupture in small abdominal aortic aneurysms. J Vasc Surg 1998; 28: 884-8.
Darling RC, Messina CR, Brewster DC, et al. Autopsy study of unoperated abdominal aortic aneurysms. The case for early resection. Circulation 1977; 56(3 Suppl): 11161-4.

[13] Thubrikar MJ, Labrosse M, Robicsek F, et al. Mechanical properties of abdominal aortic aneurysm wall. J Med Eng Tech 2001; 25: 133-42.

[14] Hirose Y, Takamiya M. Growth curve of ruptured aortic aneurysm. J Cardiovasc Surg 1998; 39: 9-13.

[15] Vorp DA, Raghavan ML, Webster MW. Mechanical wall stress in abdominal aortic aneurysm: influence of diameter and asymmetry. J Vasc Surg 1998; 27: 632-9.

[16] Scotti CM, Shkolnik AD, Muluk SC, et al. Fluid-structure interaction in abdominal aortic aneurysms: effect of asymmetry and wall thickness. Biomed Eng Online 2005; 4: 64.

[17] Doyle BJ, Callanan A, Burke PE, et al. Vessel asymmetry as an additional diagnostic tool in the assessment of abdominal aortic aneurysms. J Vasc Surg 2009; 49: 443-54.

[18] Wang DHJ, Makaroun MS, Webster MW, et al. Effect of intraluminal thrombus on wall stress in patient-specific models of abdominal aortic aneurysm. J Vasc Surg 2002; 36: 598-604.

[19] Vorp DA, Vande Geest J. Biomechanical determinants of abdominal aortic aneurysm rupture. Artherioscler Thromb Vasc Biol 2005; 25: 1558-66.

[20] Vande Geest JP, Di Martino ES, Bohra A, et al. A biomechanicsbased rupture potential index for abdominal aortic aneurysm risk assessment. Ann N Y Acad Sci 2006; 1085: 11-21.

[21] Stenbaek J, Kalin B, Swedenborg J. Growth of thrombus may be a better predictor of rupture than diameter in patients with abdominal aortic aneurysms. Eur J Vasc Endovasc Surg 2000; 20: 466-9.

[22] Giannoglu G, Giannakoulas G, Soulis J, et al. Predicting the risk of rupture of abdominal aortic aneurysms by utilizing various geometrical parameters: revisiting the diameter criterion. Angiology 2006; 57: 487-94

[23] Volokh KY, Vorp DA. A model of growth and rupture of abdominal aortic aneurysm. J Biomech 2008; 41: 1015-21.

[24] Raghavan ML, Webster MW, Vorp DA. Ex vivo biomechanical behaviour of abdominal aortic aneurysm: assessment using a new mathematical model. Ann Biomed Eng 1996; 24: 573-82.

[25] Raghavan ML, Kratzberg J, de Tolosa EMC, et al. Regional distribution of wall thickness and failure properties of human abdomina aortic aneurysm. J Biomech 2006; 39: 3010-6.

[26] Doyle BJ, Morris LG, Callanan A, et al. 3D reconstruction and manufacture of real abdominal aortic aneurysms: from CT scan to silicone model. J Biomech Eng 2008; 130: 034501-5.

[27] Morris L, Delassus P, Callanan A, et al. 3D numerical simulation of blood flow through models of the human aorta. J Biomech Eng 2005; 127: 767-75.

[28] Li Z, Kleinstreur C. A new wall stress equation for aneurysmrupture. Ann Biomed Eng 2005; 33: 209-13.

[29] Raghavan ML, Vorp DA, Federle MP, et al. Wall stress distribution on three-dimensionally reconstructed models of human abdominal aortic aneurysm. J Vasc Surg 2000; 31: 760-9.

[30] Venkatasubramaniam AK, Fagan MJ, Mehta T, et al. A comparative study of aortic wall stress using finite element analysis for ruptured and non-ruptured abdominal aortic aneurysms. Eur J Vasc Endovasc Surg 2004; 28: 168-76.

[31] Leung JH, Wright AR, Cheshire N, et al. Fluid structure interaction of patient specific abdominal aortic aneurysms: a comparison with solid stress models. Biomed Eng Online 2006; 5: 33.

[32] Papaharilaou Y, Ekaterinaris JA, Manousaki E, et al. A decoupled fluid structure approach for estimating wall stress in abdominal aortic aneurysms. J Biomech 2007; 40: 367-77.

[33] Speelman L, Bohra A, Bosboom EMH, et al. Effects of wall calcifications in patient-specific wall stress analyses of abdominal aortic aneurysms. J Biomech Eng 2007; 129: 1-5.

[34] Doyle BJ, Callanan A, McGloughlin TM. A comparison of modelling techniques for computing wall stress in abdominal aortic aneurysms. Biomed Eng Online 2007; 6: 38.

[35] Raghavan ML, Fillinger MF, Marra SP, et al. Automated methodology for determination of stress distribution in human abdominal aortic aneurysm. J Biomech Eng 2005; 127: 868-71.

[36] Wang DHG, Makaroun MS, Webster MW, et al. Mechanical properties and microstructure of intraluminal thrombus from abdominal aortic aneurysm. J Biomech Eng 2001; 123: 536-9. 
[37] Inzoli F, Boschetti F, Zappa M, et al. Biomechanical factors in abdominal aortic aneurysm rupture. Eur J Vasc Surg 1993; 7: 66774.

[38] Truijers M, Pol JA, SchultzeKool LJ, et al. Wall stress analysis in small asymptomatic, symptomatic and ruptured abdominal aortic aneurysms. Eur J Vasc Endovasc Surg 2006; 33: 401-07.

[39] Morris L, O’Donnell P, Delassus P, et al. Experimental assessment of stress patterns in abdominal aortic aneurysms using the photoelastic method. Strain 2005; 40: 165-72.

[40] Callanan A, Morris LG, McGloughlin TM. Numerical and experimental analysis of an idealised abdominal aortic aneurysm. Euro- pean Society of Biomechanics S-Hertogenbosch, Netherlands 2004.

[41] Vande Geest JP, Wang DHJ, Wisniewski SR, et al. Towards a noninvasive method for determination of patient-specific wall strength distribution in abdominal aortic aneurysms. Ann Biomed Eng 2006; 34: 1098-106.

[42] Vande Geest JP, Sacks MS, Vorp DA. The effects of aneurysm on the biaxial mechanical behaviour of human abdominal aorta. J Biomech 2006; 39: 1324-34.

[43] Rodriguez JF, Ruiz C, Doblare M, et al. Mechanical stresses in abdominal aortic aneurysms: influence of diameter, asymmetry, and material anisotropy. J Biomech Eng 2008; 130: 0210231-10.

(C) Doyle et al.; Licensee Bentham Open.

This is an open access article licensed under the terms of the Creative Commons Attribution Non-Commercial License (http://creativecommons.org/licenses/by$\mathrm{nc} / 3.0 /$ ) which permits unrestricted, non-commercial use, distribution and reproduction in any medium, provided the work is properly cited. 